\title{
UTILIZAÇÃO DA MADEIRA DE Genipa americana PARA PRODUÇÃO DE PAINÉIS DE COLAGEM LATERAL - EGP
}

\author{
Samantha Campelo ${ }^{1}$, Setsuo Iwakiri ${ }^{2}$, Rosilani Trianoski ${ }^{2}$, Osmar Romeiro de Aguiar ${ }^{3}$ \\ ${ }^{1}$ Universidade Federal do Paraná, Programa de Pós-Graduação em Engenharia Florestal, Curitiba, Paraná, Brasil - \\ samcampelo@hotmail.com \\ ${ }^{2}$ Universidade Federal do Paraná, Departamento de Engenharia e Tecnologia Florestal, Curitiba, Paraná, Brasil - setsuo@ufpr.br; \\ rosilani@ufpr.br \\ ${ }^{3}$ Universidade Federal Rural da Amazônia, Belém, Pará, Brasil - o.aguiarromeiro@gmail.com
}

Recebido para publicação: 12/09/2016 - Aceito para publicação: 22/02/2017

\begin{abstract}
Resumo
O objetivo desta pesquisa foi avaliar a viabilidade de utilização da madeira de Genipa americana para produção de painéis de colagem lateral. O material foi coletado num plantio florestal localizado na região de Tomé Açu, estado do Pará. Foram avaliadas as propriedades físicas e químicas da madeira e qualidade da colagem lateral. A colagem foi realizada com os adesivos poliacetato de vinila (PVA) e emulsão polimérica de isocianato (EPI), com diferentes gramaturas $\left(180 \mathrm{~g} / \mathrm{m}^{2}\right.$ e $\left.220 \mathrm{~g} / \mathrm{m}^{2}\right)$ e tempos de prensagem ( 3 e 4 horas). A qualidade de colagem foi avaliada por meio do ensaio de cisalhamento da linha de cola com base na norma EN 13353 e EN 13354. Os resultados indicaram que a madeira de Genipa americana possui massa especifica média de $0,607 \mathrm{~g} / \mathrm{cm}^{3}$, teor de extrativos de $6,99 \%$ e $0,61 \%$ de materiais inorgânicos. O adesivo PVA não apresentou bons resultados de colagem. A gramatura não afetou significativamente a resistência ao cisalhamento das juntas coladas com o adesivo EPI, no entanto, foi influenciada significativamente pelo tempo de prensagem. Pode-se concluir que a madeira de Genipa americana possui viabilidade técnica para produção de painéis de colagem lateral, sendo recomendado para sua produção o adesivo EPI com gramatura de $220 \mathrm{~g} / \mathrm{m}^{2}$ e tempo de prensagem de 4 horas.

Palavras-chave: Jenipapo; Emulsão polimérica de isocianato; Poliacetato de Vinila; Painéis de colagem lateral; Produtos de maior valor agregado.
\end{abstract}

\begin{abstract}
Utilization of wood of Genipa Americana L. for edge glued panel production. The objective of this research was to evaluate the feasibility of using the Genipa americana wood for the production of edge glued panels. The material was collected in a forest plantation located in the region of Tome Açu, State of Pará. There were evaluated the physical and chemical properties of the wood and the quality of edge gluing. The gluing was did with vinyl polyacetate adhesive (PVA) and polymeric emulsion of isocyanate (EPI), with different amounts $\left(180 \mathrm{~g} / \mathrm{m}^{2}\right.$ e $220 \mathrm{~g} / \mathrm{m}^{2}$ ) and pressing times ( 3 and 4 hours). The gluing quality was evaluated by glue line shear strength based in EN 13353 and EN 13354 standards. The results indicate that the wood of Genipa americana present the mean density of $0.607 \mathrm{~g} / \mathrm{cm}^{3}$, extractives content of $6,99 \%$ and inorganic materials of $0,61 \%$. PVA adhesive did not show good lbonding results. The amounts of adhesive EPI did not significantly affect the shear strength of bonded joints, however, it was significantly influenced by pressing time. It can be concluded that the wood of American Genipa has technical feasibility for the production of edge glued panels and is recommended the use of EPI adhesive with the amount of $220 \mathrm{~g} / \mathrm{m}^{2}$ and pressing time of four hours.

Keywords: Jenipapo; polymeric isocyanate emulsion; vinyl polyacetate; edge glued panels; higher valueadded products.
\end{abstract}

\section{INTRODUÇÃOO}

A exploração florestal madeireira é uma das principais atividades econômicas da região Norte do país, principalmente no estado do Pará, entretanto, nas últimas décadas este setor vem sendo alvo de fortes pressões ambientais a nível nacional e internacional, devido ao elevado índice de degradação florestal.

Atualmente, entre as principais perspectivas para o setor florestal na Amazônia, está a expansão de plantios florestais para atender a demanda de matéria-prima e a restauração de florestas primárias destruídas no passado (MARTINS et al., 2013). Os plantios florestais surgem como um excelente aliado na busca de soluções para o setor madeireiro, sendo que sob o ponto de vista econômico, são considerados investimentos ao longo prazo, dependendo da espécie plantada e do rigor do manejo dispensado. Outro aspecto importante e que deve 
ser ressaltado, é que o desenvolvimento de povoamentos florestais em regiões tropicais ocorre de forma mais rápida que em áreas temperadas.

Um dos principais requisitos das florestas plantadas para atender à demanda do setor madeireiro é possuir rápido crescimento. Nesse contexto, destaca-se a Genipa americana L., com bom crescimento. Pertencente à família Rubiaceae, e conhecida popularmente como Jenipapo ou Jenipapeiro, ocorre na América Central tropical e na América do Sul, estando presente em todo o Brasil, naturalmente ou cultivada, desde a Amazônia até São Paulo e Mato Grosso, em várias formações florestais (CARVALHO, 2003).

Em Dionísio, MG, esta espécie atingiu incremento médio anual em diâmetro de 9,90 mm por ano com casca aos 9 anos de idade (MENDES et al., 1982). Oliveira (2006) também descreve valores elevados de crescimento para espécie, onde a partir de plantios com 12 meses localizados em Brasília, DF, o incremento médio anual em diâmetro foi de $11 \mathrm{~mm}$, e o incremento médio anual em altura de $29,5 \mathrm{~cm}$.

Segundo Carvalho (2003) a Genipa americana caracteriza-se pela sua capacidade de desenvolver-se bem em solos relativamente pobres, ter preferência por terrenos úmidos, encharcados ou inundáveis, apresentar fuste reto com poucas ramificações, ter alto índice de desrama natural e ser sempreflorens. Lorenzi (2002) afirma que apesar do conhecimento do potencial produtivo e da adaptabilidade da Genipa americana nas diversas regiões tropicais, são poucos os trabalhos sobre esta espécie, principalmente em se tratando de estudos de exploração, produção e uso da sua madeira.

Entre as diversas utilizações que podem ser dadas à madeira das diferentes espécies, destacam-se os produtos de maior valor agregado, como por exemplo, portas, pisos, molduras, móveis e painéis de colagem lateral.

Os painéis de colagem lateral são painéis produzidos a partir de um conjunto de sarrafos de madeira colados lateralmente, podendo ou não apresentar colagens de topo por meio de emendas finger-joint, sendo sua adesão realizada com o auxílio de adesivos (TIENE et al., 2011). Sua produção permite a utilização de peças estreitas e curtas, com defeitos pequenos ou leves, resíduos de serrarias e de outras indústrias de base florestal, aumentando assim o rendimento por meio do reaproveitamento de um material que era descartado ou queimado como biomassa para geração de energia (DANAWADE et al., 2014).

Na produção dos painéis de colagem lateral, muitos são os fatores que podem influenciar sua qualidade final, destacando-se como principais a espécie de madeira e suas propriedades, o adesivo e as condições de colagem (IWAKIRI et al., 2015).

As espécies mais utilizadas para esta finalidade são as do gênero Pinus, especialmente o Pinus taeda, e em menor escala espécies de Eucalyptus e a Tectona grandis (ALMEIDA, 2013). Cada espécie possui características específicas, sendo a massa especifica e os extrativos as de maior influência sobre a colagem (FRIHART; HUNT, 2010).

Espécies de massa específica mais baixa proporcionam maior facilidade de colagem, pois possuem células de paredes mais finas e lumens maiores, comportando maior quantidade de adesivo, o que permite maior ancoragem, e, não requerem elevadas pressões durante a prensagem (FRIHART; HUNT, 2010). Marra (1992) complementa que a massa específica está relacionada à resistência na linha de cola, sendo que madeiras de massa específica mais elevada possuem maior resistência na linha de cola, especialmente na faixa de 0,70 a $0,80 \mathrm{~g} / \mathrm{cm}^{3}$, e acima desse valor, existe uma tendência de enfraquecimento dessa resistência.

Em relação aos extrativos, estes podem causar uma redução significativa na capacidade de adesão e contribuir para a contaminação da superfície da madeira. Os extrativos podem alterar o pH da madeira, resultando em pré-cura do adesivo ou inibindo a sua umectação, fluidez e penetração (FRIHART; HUNT, 2010). Os adesivos mais utilizados para a produção de painéis de colagem lateral são o poliacetato de vinila (PVA) e a emulsão polimérica de isocianato (EPI) (LOPES 2008; IWAKIRI et al., 2015). O adesivo PVA é conhecido popularmente como "cola branca", apresenta alta resistência mecânica em ambiente seco, porém limitações de uso em ambientes com altas temperaturas e umidade relativa, é de baixo custo, inodoro, fácil manuseio, rápida secagem, pouco abrasivo e flexível. Já o EPI é um adesivo bi componente, de alta resistência à alta temperatura, à água e à solventes, boa estabilidade, cura rápida, flexível, porém de maior custo (FRIHART; HUNT, 2010).

Cada adesivo requer parâmetros específicos para promover uma colagem de qualidade, podendo se destacar a gramatura e o tempo de prensagem. Segundo Fonte e Trianoski (2015), quando a gramatura aplicada é menor que a ideal ou em demasia, a resistência do produto final é consideravelmente alterada. Gramaturas muito baixas implicam em resistência inferior devido à aderência e ancoragem insuficientes. Já gramaturas elevadas geram o aumento do tempo em aberto e de prensagem, e podem ainda, promover o desperdício pelo derramamento do adesivo em excesso pelas bordas dos painéis, resultando em prejuízos econômicos (MARRA, 1992; IWAKIRI et al., 2014). Segundo Lopes (2008), a faixa de gramatura mais utilizada pela indústria varia de 180 a $220 \mathrm{~g} / \mathrm{m}^{2}$.

Em relação ao tempo de prensagem, existe um tempo mínimo necessário para que ocorram as funções de mobilidade do adesivo na interface com a madeira e posterior secagem e cura do mesmo. Baixos tempos de prensagem podem não ser suficientes para que todas as reações do adesivo ocorram sob pressão, gerando uma 
linha de cola de baixo desempenho, e elevados tempos de permanência do painel na prensa podem implicar em redução da produtividade e aumento no consumo de energia (IWAKIRI et al., 2014).

Neste contexto, considerando, a importância de estudos com madeiras de espécies pouco conhecidas tecnologicamente, a importância do painel de colagem lateral para o setor madeireiro e para a economia, e os parâmetros utilizados no processo produtivo deste produto, esta pesquisa tem como objetivo avaliar a viabilidade de utilização da madeira de Genipa americana para produção de painéis de colagem lateral.

\section{MATERIAIS E MÉTODOS}

Para a realização deste trabalho foram utilizadas três árvores de Genipa americana coletadas em um plantio florestal com nove anos de idade, localizado na região de Tomé Açu no estado do Pará, de propriedade da Embrapa Amazônia Oriental. As árvores foram selecionadas levando em consideração o fuste reto e boas condições fitossanitárias, sendo posteriormente abatidas e seccionadas para obtenção das toras e discos na região do DAP.

Os discos foram convertidos em cunhas e peças retangulares e posteriormente utilizados nos ensaios da massa específica básica e aparente, e das propriedades químicas. A determinação da massa específica básica e aparente seguiu os procedimentos da norma Copant 461 (1972). As propriedades químicas foram avaliadas de acordo com a metodologia proposta pelas normas TAPPI, sendo quantificados o teor de extrativos totais (TAPPI 204:1997) e os materiais inorgânicos (TAPPI 211:2002).

As toras foram desdobradas em tábuas, classificadas visualmente quanto à ocorrência de nós, rachaduras e podridão, secas em estufa convencional e climatizadas até teor de umidade de aproximadamente $12 \%$. Após a secagem e climatização, foram convertidas em sarrafos com dimensões de $310 \times 55 \times 25 \mathrm{~mm}$, sendo selecionados para a colagem das juntas, sarrafos com as duas faces limpas ou isentas de defeitos. Não houve classificação dos sarrafos quanto ao plano de corte.

Os adesivos utilizados na colagem dos sarrafos foram o Poliacetado de vinila (PVA) e a Emulsão polimérica de isocianato (EPI). Avaliou-se o efeito do tipo de adesivo sobre a qualidade de colagem, assim como, o efeito da gramatura e o tempo de prensagem do adesivo EPI, conforme delineamento experimental proposto na tabela 1 .

Os adesivos foram aplicados em uma das superfícies do sarrafo e espalhados com um rolo de espuma. A uniformidade de distribuição foi monitorada visualmente e o controle da gramatura foi realizado em uma balança digital ( $2.000 \mathrm{~g})$ com precisão de $0,01 \mathrm{~g}$. Após a aplicação do adesivo, as juntas (4 repetições por tratamento) foram submetidas a prensagem a temperatura ambiente, utilizando-se pressão específica de $6,45 \mathrm{kgf} / \mathrm{cm}^{2}$ exercida por meio de um torquímetro, e mantidas sob pressão pelos tempos pré-estabelecidos em cada tratamento.

As juntas coladas foram climatizadas à temperatura de $20 \pm 2{ }^{\circ} \mathrm{C}$ e umidade relativa de $65 \pm 5 \%$, sendo então, confeccionados os corpos de prova para avaliação da qualidade de colagem por meio do ensaio de cisalhamento da linha de cola. Os ensaios foram conduzidos de acordo com a metodologia descrita pela norma EN 13354:2008, em estado seco e úmido (24 horas de imersão em água a $20{ }^{\circ} \mathrm{C}$ ), e os resultados foram comparados com os requisitos mínimos estabelecidos na norma EN 13353:2008. Após o ensaio de cisalhamento foram avaliadas também, as percentagens de falha na madeira na superfície de ruptura da linha de cola, segundo as recomendações da norma EN 314 (2002).

Os resultados da qualidade das juntas coladas foram submetidos à análise estatística por meio dos testes de Grubs, Shapiro Wilks, Bartlet, Análise de variância e comparação de médias de Tukey. A comparação entre os adesivos PVAc e EPI foi realizada por meio da análise de variância simples, e o efeito da gramatura e do tempo de prensagem foi avaliada a partir da análise de variância em arranjo fatorial (2x2). Todos os testes foram realizados no programa Statgraphics XV a 95\% de confiabilidade.

Tabela 1. Plano experimental.

Table 1. Experimental plan.

\begin{tabular}{cccc}
\hline Tratamento & Adesivo & $\begin{array}{c}\text { Gramatura } \\
\left(\mathbf{g} / \mathbf{m}^{\mathbf{2}}\right)\end{array}$ & $\begin{array}{c}\text { Tempo de prensagem } \\
(\mathbf{h})\end{array}$ \\
\hline \multicolumn{4}{c}{ Efeito do adesivo } \\
\\
\hline 1 & PVA & 180 & 3 \\
2 & EPI & 180 & 3 \\
\hline 3 & Efeito da gramatura e do tempo de prensagem sobre o adesivo EPI \\
4 & EPI & 180 & 3 \\
5 & EPI & 180 & 4 \\
6 & EPI & 220 & 3 \\
\hline
\end{tabular}

PVA: Poliacetato de Vinila; EPI: Emulsão Polimérica de Isocianato.

FLORESTA, Curitiba, PR, v. 47, n. 1, p. 129 - 135, jan. / mar. 2017.

Campelo, S. et al.

ISSN eletrônico 1982-4688

DOI: $10.5380 /$ rf.v47i1.48453 


\section{RESULTADOS}

\section{Propriedades da madeira}

Os resultados apresentados na tabela 2 indicam que a madeira de Genipa americana possui massa especifica básica de $0,607 \mathrm{~g} / \mathrm{cm}^{3}$, sendo classificada como média de acordo com Melo et al. (1990), que relatam que esta classe apresenta valores de 0,510 a $0,720 \mathrm{~g} / \mathrm{cm}^{3}$.

Os resultados obtidos para a massa específica deste estudo são similares aos de Mendes et al. (1982), que obtiveram valor de $0,66 \mathrm{~g} / \mathrm{cm}^{3}$ para massa específica básica, e ao citado por Lorenzi (2002) de $0,68 \mathrm{~g} / \mathrm{cm}^{3}$.

Tabela 2. Valores médios das propriedades físico-químicas da madeira de Genipa americana.

Table 2. Average values of the physical and chemical properties of wood of Genipa Americana.

\begin{tabular}{lc}
\hline Propriedade & Valor médio \\
\hline Massa específica básica $\left(\mathrm{g} / \mathrm{cm}^{3}\right)$ & $0,607(4,75)$ \\
Massa específica aparente $\left(\mathrm{g} / \mathrm{cm}^{3}\right)$ & $0,738(6,03)$ \\
Extrativos totais $(\%)$ & $6,99(2,20)$ \\
Materiais inorgânicos $(\%)$ & $0,61(7,20)$ \\
\hline Valores entre parênteses referem-se ao coeficiente de variação das amostras em percentual.
\end{tabular}

A madeira de Genipa americana apresentou valor médio de extrativos totais de 6,99\%, o qual, é superior à faixa de variação descrita por Fengel e Wegener (2003), que é de 1\% a 5\% para folhosas; no entanto, está compatível com Sjöström (1981) que relata que o teor destes componentes é normalmente inferior a $10 \%$.

Em relação ao teor de materiais inorgânicos, foi observado valor médio de $0,61 \%$, valor relativamente baixo quando comparado aos relatados por Sjöström (1981) e Fengel e Wegener (2003), que afirmam que em espécies de clima tropical esse componente pode atingir até $5 \%$.

\section{Propriedades dos painéis}

Efeito do tipo de adesivo

Na tabela 3 estão apresentados os valores médios da resistência ao cisalhamento, $5^{\circ}$ percentil inferior e percentual de falha na madeira das juntas coladas de Genipa americana com os adesivos PVA e EPI.

Tabela 3. Valores médios da resistência ao cisalhamento das juntas coladas de Genipa americana com os adesivos PVA e EPI

Table 3. Average values of the shear strength of glued joints of Genipa Americana with PVA and EPI adhesives.

\begin{tabular}{lcccccc}
\hline \multirow{2}{*}{ Tratamento } & \multicolumn{3}{c}{ Seco } & \multicolumn{3}{c}{ Umido } \\
\cline { 2 - 7 } & $\begin{array}{c}\text { Cisalhamento } \\
(\mathbf{M P a})\end{array}$ & $\begin{array}{c}\mathbf{5}^{\circ} \text { Perc. } \\
(\mathbf{M P a})\end{array}$ & $\begin{array}{c}\text { Falha } \\
(\boldsymbol{\%})\end{array}$ & $\begin{array}{c}\text { Cisalhamento } \\
(\mathbf{M P a})\end{array}$ & $\begin{array}{c}\mathbf{5}^{\circ} \text { Perc. } \\
(\mathbf{M P a})\end{array}$ & $\begin{array}{c}\text { Falha } \\
(\boldsymbol{\%})\end{array}$ \\
\hline $\mathrm{PVA}-180 \mathrm{~g} / \mathrm{m}^{2} / 3 \mathrm{~h}$ & $2,81 \mathrm{~b}(44,25)$ & 1,15 & 0 & $0,28 \mathrm{~b}(58,65)$ & 0,10 & 0 \\
\hline $\mathrm{EPI}-180 \mathrm{~g} / \mathrm{m}^{2} / 3 \mathrm{~h}$ & $6,43 \mathrm{a}(51,47)$ & 2,29 & 9 & $4,20 \mathrm{a}(66,88)$ & 0,57 & 3 \\
\hline
\end{tabular}

Médias seguidas de mesma letra na mesma coluna são estatisticamente iguais pelo teste de Tukey a 95\% de confiabilidade; Valor es entre parênteses referem-se ao coeficiente de variação das amostras, em percentual.

Tanto para o teste seco, quanto para o úmido, foram observadas diferenças estatísticas significativas entre os dois adesivos utilizados, sendo que o adesivo EPI apresentou melhor desempenho que o PVA, quanto a qualidade de colagem da madeira de Genipa americana. Apesar do melhor desempenho em termos de resistência, mesmo o adesivo EPI não atingiu o requisito mínimo de $2,50 \mathrm{MPa}$ para o $5^{\circ}$ percentil inferior em nenhuma das condições de ensaio, bem como baixos percentuais de falha na madeira, indicando desta forma, necessidade de alteração de variáveis do processo de colagem.

Efeito da gramatura e tempo de prensagem

Na tabela 4 estão apresentados os valores médios dos ensaios de cisalhamento das juntas coladas com o adesivo EPI em diferentes gramaturas e tempos de prensagem.

Foram constatadas diferenças estatísticas significativas no ensaio de cisalhamento a seco, sendo que os tratamentos colados com gramatura de $220 \mathrm{~g} / \mathrm{m}^{2}$ e prensados com os tempos de $3 \mathrm{~h}$ e $4 \mathrm{~h}$, e o tratamento colado com gramatura de $180 \mathrm{~g} / \mathrm{m}^{2}$ e $4 \mathrm{~h}$ apresentaram-se estatisticamente iguais entre si, e superiores em relação ao tratamento produzido com gramatura de $180 \mathrm{~g} / \mathrm{m}^{2}$ e prensado por $3 \mathrm{~h}$. Observou-se ainda, que o último tratamento mencionado, foi o único que não atingiu o requisito mínimo de $2,50 \mathrm{MPa}$ para o $5^{\circ}$ percentil inferior nesta condição. 
Tabela 4. Valores médios da resistência ao cisalhamento das juntas coladas de Genipa americana com o adesivo EPI em diferentes gramaturas e tempos de prensagem.

Table 4. Average values of the shear strength of glued joints of Genipa Americana with EPI adhesive in different amount and pressing times.

\begin{tabular}{ccccccc}
\hline \multirow{2}{*}{ Tratamento } & \multicolumn{3}{c}{ Seco } & \multicolumn{3}{c}{ Umido } \\
\cline { 2 - 7 } & $\begin{array}{c}\text { Cisalhamento } \\
(\mathbf{M P a})\end{array}$ & $\begin{array}{c}\mathbf{5}^{\circ} \text { Perc. } \\
(\mathbf{M P a})\end{array}$ & $\begin{array}{c}\text { Falha } \\
(\boldsymbol{\%})\end{array}$ & $\begin{array}{c}\text { Cisalhamento } \\
(\mathbf{M P a})\end{array}$ & $\begin{array}{c}\mathbf{5}^{\circ} \text { Perc. } \\
(\mathbf{M P a})\end{array}$ & $\begin{array}{c}\text { Falha } \\
(\boldsymbol{\%})\end{array}$ \\
\hline EPI $-180 \mathrm{~g} / \mathrm{m}^{2} / 3 \mathrm{~h}$ & $6,43 \mathrm{~b}(51,47)$ & 2,29 & 9 & $4,20 \mathrm{a}(66,88)$ & 0,72 & 2 \\
EPI - $220 \mathrm{~g} / \mathrm{m}^{2} / 3 \mathrm{~h}$ & $7,36 \mathrm{a}(27,06)$ & 4,54 & 17 & $4,51 \mathrm{a}(69,95)$ & 0,82 & 5 \\
EPI - $180 \mathrm{~g} / \mathrm{m}^{2} / 4 \mathrm{~h}$ & $7,81 \mathrm{a}(34,74)$ & 4,47 & 12 & $5,30 \mathrm{a}(43,03)$ & 2,25 & 3 \\
EPI - $220 \mathrm{~g} / \mathrm{m}^{2} / 4 \mathrm{~h}$ & $7,77 \mathrm{a}(38,22)$ & 3,25 & 24 & $4,81 \mathrm{a}(42,98)$ & 2,84 & 6 \\
\hline
\end{tabular}

Médias seguidas de mesma letra na mesma coluna são estatisticamente iguais pelo teste de Tukey a 95\% de confiabilidade; Valores entre parênteses referem-se ao coeficiente de variação das amostras, em percentual.

No ensaio úmido não foram constatadas diferenças estatísticas significativas entre as resistências ao cisalhamento dos tratamentos propostos. Por outro lado, apenas o tratamento colado com gramatura de $220 \mathrm{~g} / \mathrm{m}^{2}$ e $4 \mathrm{~h}$ de prensagem atingiu o valor mínimo de 2,50 MPa para o $5^{\circ}$ percentil inferior, conforme a norma EN 13353 (2008).

Na tabela 5 estão apresentados os resultados da análise fatorial dos efeitos da gramatura e do tempo de prensagem sobre a qualidade de colagem da madeira de Genipa americana.

Tabela 5. Efeito da gramatura e do tempo de prensagem sobre a qualidade de colagem da madeira de Genipa americana com adesivoEPI.

Table 5. Effect of the amount and pressing time on the bonding qulity of wood of Genipa Americana with EPI adhesive.

\begin{tabular}{|c|c|c|c|c|c|c|}
\hline \multirow[b]{2}{*}{ Tratamento } & \multicolumn{3}{|c|}{ Seco } & \multicolumn{3}{|c|}{ Úmido } \\
\hline & $\begin{array}{l}\text { Cisalhamento } \\
(\mathrm{MPa})\end{array}$ & $\begin{array}{l}5^{\circ} \text { Perc. } \\
(\mathrm{MPa})\end{array}$ & Falha $(\%)$ & $\begin{array}{c}\text { Cisalhamento } \\
(\mathrm{MPa})\end{array}$ & $\begin{array}{c}5^{\circ} \text { Perc. } \\
(\mathrm{MPa})\end{array}$ & Falha $(\%)$ \\
\hline \multicolumn{7}{|c|}{ Gramatura } \\
\hline $180 \mathrm{~g} / \mathrm{cm}^{2}$ & 7,16 a $(42,43)$ & 2,44 & 11 & 5,04 a $(48,19)$ & 1,16 & 3 \\
\hline $220 \mathrm{~g} / \mathrm{cm}^{2}$ & 7,56 a $(32,84)$ & 3,49 & 21 & $4,87 \mathrm{a}(52,62)$ & 0,82 & 6 \\
\hline \multicolumn{7}{|c|}{ Tempo } \\
\hline $3 \mathrm{~h}$ & $6,92 \mathrm{~b}(38,8)$ & 2,44 & 13 & $4,60 \mathrm{~b}(32,90)$ & 0,78 & 4 \\
\hline $4 \mathrm{~h}$ & 7,79 a $(35,88)$ & 3,55 & 18 & 5,32 a $(37,35)$ & 2,53 & 5 \\
\hline
\end{tabular}

Médias seguidas de mesma letra na mesma coluna são estatisticamente iguais pelo teste de Tukey a 95\% de confiabilidade; Valores entre parênteses referem-se ao coeficiente de variação das amostras, em percentual.

Não foram observadas diferenças estatísticas significativas para a variável gramatura em ambas as condições de ensaio. Apenas para a gramatura $220 \mathrm{~g} / \mathrm{m}^{2}$ avaliada em estado seco atingiu o valor de $2,50 \mathrm{MPa}$ para o $5^{\circ}$ percentil inferior. Observou-se também, baixos valores médios de falha na madeira, sendo que o aumento do adesivo proporcionou um ligeiro aumento nesta variável.

$\mathrm{O}$ efeito do tempo de prensagem mostrou-se estatisticamente significativo, sendo que o tempo de 4 horas apresentou valores médios de resistência ao cisalhamento superiores em relação ao tempo de prensagem de 3 horas. O tempo de prensagem de 4 horas atingiu o requisito mínimo de $2,50 \mathrm{MPa}$ para o $5^{\circ}$ percentil inferior, tanto no ensaio seco quanto úmido, estando, portanto, aprovado segundo a norma EN 13353 (2008).

\section{DISCUSSÕES}

Espécies de madeiras de baixa ou média massa específica são relativamente mais fáceis de serem coladas quando comparadas com espécies de alta massa especifica, pelo fato de possuírem paredes celulares mais finas e lumens maiores, o que facilita a mobilidade do adesivo na estrutura lenhosa, além de comportarem maior quantidade de adesivo, permitindo maior ancoragem. Marra (1992) corrobora que quanto mais densa for a madeira, menor será sua permeabilidade, podendo gerar uma ligação adesiva mais superficial e provavelmente mais fraca.

Fengel e Wegener (2003), afirmam que, elevados teores de extrativos na madeira contribuem para contaminação de sua superfície, podem alterar o $\mathrm{pH}$, influenciando negativamente o processo de colagem por meio da pré-cura, e, ainda, podem gerar umectação e fluidez insuficientes.

De acordo com Sjöström (1981), baixos valores de materiais inorgânicos são desejáveis em madeiras utilizadas para a produção de painéis EGP, pois antes do processo de colagem, o material precisa ser dimensionado em serras e plainas, e quanto menor o teor destes componentes, menor é o desgaste das ferramentas de corte. 
Avaliações comparativas dos efeitos do adesivo EPI quando comparada com o adesivo PVA pode ser explicada pela sua composição química ou formulação. Ambos os adesivos apresentam em sua composição acetato de vinila, no entanto, no PVA, o acetato é polimerizado em uma emulsão aquosa formando uma dispersão aquosa de Poliacetato de vinila, e no EPI, o acetato de vinila é combinado com um isocianato polimérico (difenilmetano-diisocianato). De acordo com Clemente et al. (2014) os diisocianatos são responsáveis pelo chamado segmento rígido e conferem aos polímeros propriedades de dureza, resistência ao cisalhamento, e módulo de elasticidade à tração e/ou compressão.

Além da composição química, o melhor desempenho do adesivo EPI também pode ser justificado por suas propriedades intrínsecas de adesão à madeira. A cura deste adesivo ocorre pela reação de seus grupos de isocianato com os grupos hidroxilas da madeira, promovendo a adesão química. Em contrapartida, a cura do adesivo PVAc ocorre pela perda de água e união da sua estrutura química (moléculas de acetato de vinila), caracterizando-se por uma colagem menos resistente.

Resultados similares foram encontrados por Iwakiri et al. (2015), que avaliando a qualidade de colagem da madeira de Cryptomeria japonica e Sequoia sempervirens com os adesivos PVA e EPI, obtiveram resistência estatisticamente superior para o adesivo EPI, e por Almeida (2013), que em estudo da viabilidade de utilização de resíduos de madeiras tropicais para produção de painéis EGP, também obteve melhor desempenho das espécies avaliadas a partir da colagem com adesivo EPI.

Em comparação com a literatura, verifica-se que a madeira de Genipa americana colada com adesivo EPI apresentou melhor desempenho em relação às espécies Tabebuia spp., Hymenaea spp., Astronium lecointei e Couratari spp, e desempenho inferior quando comparada com as espécies Pterogyne nitens e Patagonula sp, todas estudadas por Almeida (2013) e também coladas com este adesivo. Por outro lado, os resultados obtidos nesta pesquisa foram inferiores quando comparados com duas espécies de coníferas (Cryptomeria japônica e Sequoia sempervirens) estudadas por Iwakiri et al. (2015), que obtiveram valores de resistência ao cisalhamento de 7,10 MPa a 10,51 MPa e $5^{\circ}$ percentil inferior de 5,91 MPa a 8,22 $\mathrm{MPa}$ no ensaio seco, e de 4,26 $\mathrm{MPa}$ a 5,92 $\mathrm{MPa}$ e de 3,30 MPa a 5,35 MPa, respectivamente, no ensaio úmido.

Embora a gramatura seja um parâmetro que influencia diretamente a colagem, sendo que quanto maior esta variável maior é a resistência e melhor é a qualidade do painel, a variação de gramatura de $180 \mathrm{~g} / \mathrm{m}^{2}$ para $220 \mathrm{~g} / \mathrm{m}^{2}$ utilizada neste estudo não mostrou claramente este efeito. Relações similares foram constatados por Iwakiri et al. (2015) e por Fonte e Trianoski (2015).

Os melhores resultados de resistência ao cisalhamento e $5^{\circ}$ percentil inferior apresentados com o tempo de prensagem de 4 horas podem estar relacionados a melhor umectação e maior cura do adesivo sob pressão. Segundo Marra (1992) existe um tempo mínimo necessário para que ocorram as funções de mobilidade do adesivo na interface com a madeira e posterior secagem e cura do mesmo.

\section{CONCLUSÕES}

- As propriedades físico-químicas da madeira de Genipa americana indicaram que a espécie possui massa especifica média e teor de extrativos e materiais inorgânicos compatíveis com a literatura.

- $\quad$ O adesivo a base de emulsão polimérica de isocianato (EPI) apresentou qualidade de colagem superior em relação ao poliacetato de vinila (PVA).

- A gramatura não exerceu influência significativa sobre a qualidade de colagem da madeira de Genipa americana, podendo ser empregada a gramatura menor devido a melhor relação custo benefício.

- O tempo de prensagem afetou significativamente a qualidade de colagem, sendo que o tempo de 4 horas é mais indicado para a colagem da madeira de Genipa americana.

- A madeira de Genipa americana possui viabilidade técnica para produção de painéis de colagem lateral não estruturais utilizados em ambientes secos, sendo recomendado para sua produção o adesivo EPI com gramatura de $220 \mathrm{~g} / \mathrm{m}^{2}$ e tempo de prensagem de 4 horas.

\section{REFERÊNCIAS}

ALMEIDA, V. C. Avaliação do potencial de uso de resíduos de madeira tropical para produção de painéis colados lateralmente - EGP. 122 f. Tese (Doutorado em Engenharia Florestal) - Universidade Federal de Paraná, Curitiba, 2013.

CARVALHO, P. E. R. Jenipapeiro. Colombo: Embrapa (Embrapa. Circular Técnica, 80), 2003, 14 p.

CLEMENTE, M.; ROCHA, R. J.; IHA, K.; ROCCO J. A. A. F. Desenvolvimento de tecnologia de pré-polímeros na síntese de poliuretanos empregados em combustíveis sólidos. Química Nova, v. 37, n. 6, p. 982-988, 2014.

COMISSÃO PANAMERICANA DE NORMAS TÉCNICAS - COPANT. COPANT 461. Determinación Del 
peso especifico aparente. 1972.

DANAWADE, B. A.; MALAGI, R. R.; PATIL, B. S.; HANAMAPURE R. S. Effect of finger joint on flexural strength of teak wood. International Journal of Engineering and Technology, v. 5, n. 6, p. 4929-4937, 2014.

EUROPEAN COMMITTEE FOR STANDARDIZATION - CEN. EN 314-2. Contraplacado: Qualidade de Colagem - Requisitos. Brussels. 2002.

EN 13353. Solid wood panels (SWP) - Requirements - 11 p. Bruxelas, 2003.

EN 13354. Solid wood panels - Bonding quality - Test Method - 11 p. Bruxelas, 2003.

FENGEL, D.; WEGENER, G. Wood Chemistry, Ultrastructure, Reactions. Berlin/New York: Walter de Gruyter, 2003, $400 \mathrm{p}$.

FONTE, A. P. N.; TRIANOSKI, R. Efeito da gramatura sobre a qualidade de colagem lateral da madeira de Tectona grandis. Revista de Ciências Agroveterinárias, Lages, v. 14, n. 3, p. 224-233, 2015.

FRIHART, C. R.; HUNT, C. G. Adhesives with Wood Materials Bond Formation and Performance. Wood Handbook - Wood as an engineering material, Madison, cap. 10, p. 1-24, 2010.

IWAKIRI, S.; TRIANOSKI, R.; FRANÇA, R. F.; GONÇALVES, T. A. P.; LOIOLA, P. L.; CAMPELO, S. R. F.; FARIAS, S. M. de A. P. Avaliação da resistência de juntas coladas da madeira de Cryptomeria japonica e Sequoia sempervirens com diferentes adesivos. Scientia Forestalis, Piracicaba, v. 43, n. 105, p. 19-26, 2015.

IWAKIRI, S.; MATOS, J. L.M.; PRATA, J. G.; TRIANOSKI, R., PARCHEN, C. F., CASTRO, V. G.; IWAKIRI, V. T. Características de vigas laminadas coladas confeccionadas com madeira de teca (Tectona grandis). Floresta e Ambiente, Curitiba, v. 21, v. 2, 269-275, 2014.

LOPES, M. C. Espectroscopia no infravermelho próximo aplicada na avaliação de painéis de madeira colados lateralmente. 131 f. Tese (Doutorado em Engenharia Florestal) - Setor de Ciências Agrárias, Universidade Federal do Paraná, Curitiba, 2008.

LORENZI, H. Árvores brasileiras: manual de identificação e cultivo de plantas arbóreas nativas do Brasil. Nova Odessa: Instituto Plantarum, 2002.

MARRA, A. A. Technology of wood bonding: Principles in practice. New York: Van Nostrand Reinhold, $1992,453 \mathrm{p}$.

MARTINS, H. F. A.; FONSECA, A.; SOUZA, C.; SALES, M. Boletim transparência florestal da Amazônia legal. Belém: Imazon, 2013. 12 p.

MELO, J. E.; CORADIN, V. T. R.; MENDES, J. C. Classes de densidade para madeiras da Amazônia Brasileira. In: $6^{\circ}$ Congresso florestal Brasileiro, Florestas e Meio ambiente: Conservação e Produção, patrimônio social. Anais. Campos do Jordão -SP, 1990, p. 695-699.

MENDES, C. J.; REZENDE, G. C. de.; SUITER FILHO, W.; MORAES, T. S. de A. Considerações sobre o potencial silvicultural e energético de quatro espécies nativas. In: CONGRESSO NACIONAL SOBRE ESSÊNCIAS NATIVAS, 1982, Campos do Jordão. Anais. São Paulo: Instituto Florestal, 1982. p. 1354-1359.

OLIVEIRA, F. F. Plantio de espécies nativas e uso de poleiros artificiais na restauração de uma área perturbada de cerrado sentido restrito em ambiente urbano no Distrito Federal, Brasil. 90 f. Dissertação (Mestrado em Ecologia) - Universidade de Brasília, Brasília, 2006.

SJÖSTRÖM, E. Wood Chemistry fundamentals and applications. New York: Academic Press, 1981, 223p.

TECHNICAL ASSOCIATION OF THE PULP AND PAPER INDUSTRY - TAPPI. 1997. TAPPI 204. Solvent extractives of wood and pulp. Atlanta, 2002.

TAPPI 211. Ash in wood, pulp, paper and paperboard: combustion at $525^{\circ} \mathrm{C}$. Atlanta, 2002.

TIENNE, D. L. D. C.; NASCIMENTO, A. M. do; GARCIA, R. A.; SILVA, D. B. Qualidade de adesão de juntas de madeira de Pinus coladas em condições simuladas de serviço interna e externa. Floresta e Ambiente, Seropédica, v. 18, n. 1, p. 16-29, 2011.

TRIANOSKI, R.; IWAKIRI, S.; MATOS, J. L. M.; HIGA, A. R.; BRAZ, R. L. Avaliação das propriedades de painéis compensados de Melia azedarach L. produzidos com diferentes gramaturas e tempos de prensagem. Árvore, Viçosa, 39(4), 759-768, 2015. 